\title{
Quintessential Conceptualization of Bone Graft
}

\author{
Shipra Jaiswal' ${ }^{1}$, Amit Wadhawan ${ }^{2}$, Mehvish Saleem ${ }^{3}$ \\ ${ }^{1}$ Post Graduate Student, Department of Periodontology, Subharti Dental College And Hospital,Swami \\ Vivekanand Subharti University Meerut, ${ }^{2}$ Professor, Department of Periodontology, Subharti Dental College and \\ Hospital,Swami Vivekanand Subharti University Meerut, ${ }^{3}$ Senior Lecturer, Department of Periodontology, Subharti \\ Dental College and Hospital, Swami Vivekanand Subharti University Meerut
}

\begin{abstract}
As we know that healthy periodontium, is affected in most of the periodontal diseases. Which further leads to damage of hard and soft tissues. To attain back the lost structures the use of bone grafts have shown promising results. Managed either by mechanically recontouring it or by grafting techniques. Replacement grafts are widely accepted as bone formation by promoting for periodontal regeneration . There are many bone graft material which are used as bone substitute to fill the defect site the graft material acts like scaffold on which body react it by certain bone mechanism helps to regenerate new bone. This article give an overview on history, rationale and objectives of bone replacement grafts, Biology of bone healing, Indications for bone grafting include, Ideal requirements of bone grafts,List of bone graft according to origin.
\end{abstract}

Keywords: Alloplast, bone allograft, bone autograft, periodontal regeneration, xenograft

\section{Introduction}

Modern periodontics aims to maintain the healthy teeth and their supporting structures and the main goal is to control the infection and regenerate the lost supporting structures. Replacement of bone grafts are widely accepted as bone formation by promoting for periodontal regeneration. There are many bone graft material which are used as bone substitute to fill the defect site the graft material acts like scaffold on which body react it by certain bone mechanism helps to regenerate new bone. The basic principle of tissue regeneration is to stimulate a cascade of wound healing events. Hence, this review article focuses on the concept of bone graft discussing about various bone graft materials available and through light on the recent bone grafts. ${ }^{1}$

History of Bone Grafts: Historically, autogenous grafts were the first bone replacement grafts to be reported

\footnotetext{
Corresponding Author:

Dr. Shipra Jaiswal

Post Graduate Student, Department of Periodontology,

Subharti Dental College and Hospital, Swami

Vivekanand Subharti University-250005

e-mail: jaiswallshipra@gmail.com
}

for periodontal application. Allogeneic freeze-dried bone allografts were first introduced to periodontics in the early 1970's, while Demineralized freeze-dried bone allografts gained wider application in the late 1980's with an even increasing market share in 1990 's. ${ }^{2}$ Way back bone grafts used for reconstructing intraosseous by Hegedus in $1923 .{ }^{3}$ further revived in 1965 by Nabers and O'leary, they used shavings of cortical bone removed by hand chisels during osteoplasty and ostectomy and they were used to treat one, two-wall defect. ${ }^{4}$ Allografts of iliac bone and marrow were used by Schallhorn et al. in $1970 .^{5}$ The era of hydroxyapatite (HA) in regenerative science dates back to 1950 s when bioceramics were used as an inert scaffold for filling of the bone defects. ${ }^{6}$ Yukna in 1990 did a clinical 6-month study on hard tissue replacement (HTR) polymer (HTR synthetic bone) and showed a significant defect fill and improved attachment level relative to open flap debridement (OFD). ${ }^{7}$ Bio-Oss is a bovine bone from which all inorganic components are removed and used for regeneration by Richardson et al. in $1999 .{ }^{8}$

Rationale and Objectives of Bone Replacement Grafts: Periodontal therapy is directed not only at inflammation control but also at pocket reduction and correction of associated bone defects. When applicable, 
regeneration of the lost bone and periodontal attachment improves the support of the tooth and hopefully its longterm prognosis. At the present time, bone replacement grafts are the only modality of therapy for which there is histologic evidence, in humans, of regeneration of new attachment composed of new bone, cementum and periodontal ligament coronal to the base of a previous osseous defect ${ }^{9}$.

\section{Clinical Objectives of Bone Grafting:}

The objectives of bone grafting procedure in patients with periodontitis are as follows ${ }^{10}$ :

1. Probing depth reduction

2. Clinical attachment gain

3. Bone fill of osseous defects and

4. Regeneration of new bone

5. Establish a healthy maintainable environment.

Numerous case reports and controlled clinical trials have provided clinical data on achieving histologic analysis, however, can accurately determine whether any true periodontal regeneration has developed because this is the onlymethod that reveals the type of wound healing that occurs next to the root surfaces after grafting is undertaken. ${ }^{11}$

\section{Ideal characteristics of bone graft are as follows:}

1. Nontoxic

2. Non-antigenic

3. Resistant to infection

4. No root resorption

5. Strong and resilient

6. Easily adaptable

7. Readily and sufficiently available

8. Minimal surgical procedure

9. Stimulates new attachment

Based on these scientifically acceptable criteria,New attachment apparatus has been observed after certain autogenous and allogeneic grafts. ${ }^{12}$

\section{Biology of Bone Healing:}

1. Actively forms new bone

2. Induces bone formation
3. Creates passive surface for bone formation

4. Provides mechanical obstruction

The transplantation of bone or biosynthetic materials to repair skeletal defects is now an accepted surgical technique Materials reported to have been used include cancellous, cortical, and corticocancellous bone, osteochondral plugs or synthetic material. Bone grafts provide osteogenic potential (primary osteogenesis or (osteoinduction) and scaffolding for in growth of new elements (osteoconduction). ${ }^{13}$ Bone has the unique ability to heal completely and regain its original structure and mechanical properties; repaired tissue consists of new bone rather than scar tissue. The net biologic activity of the graft is the sum of its inherent biologic activity, its capacity to activate surrounding host tissues and its ability to support the in growth of host osteogenic tissue. During incorporation the graft site goes through several concurrent phases. ${ }^{14}$ Bone graft success depends on the host recipient site, local growth factors of the host bed, bone graft viability, the volume of bone grafted and the structural function of the bone graft. Motion at the graft/host bone or soft tissue interface will impede or prevent revascularization. ${ }^{15}$

- The use of bone grafting or replacement materials is based on these materials may facilitate formation of alveolar bone, periodontal ligament and root cementum through one of the following mechanisms ${ }^{16}$ :

1. Contain bone forming cells (osteoneogenesis).

2. Serve as scaffold for bone formation (osteoconduction).

3. Contain bone-inducing substances (osteoinduction).

\section{Indications:}

- Deep intraosseous defects of varying morphology

- Shallow intraosseous defects

- Furcations and shallow wide crater defect

- Ridge augmentation

- Combined procedure with GTR

- Peri-implant regenerative therapy.

\section{Ideal Requirements of Bone Grafts}

1. Should be immunologically acceptable

2. Should be resistant to infection

3. Should be biocompatible 
4. Should be easily available

5. Should be cost effective

\section{Bone Graft According to Origin:}

Autogenous Grafts: Autogenous bone graft, which is harvested from the patient's own body, is considered ideal because of its osteoconductive and osteoinductive properties and because it contains a source of osteoprogenitor cells. It is still considered the gold standard among graft materials because they are superior at retaining cell viability. It combines all properties required in a bone graft material: osteoinduction (bone morphogenetic proteins (BMPs) and other growth factors), osteogenesis (osteoprogenitor cells) and osteoconduction (scaffold). Autogenous type of graft is harvested either from intraoral or extraoral donor sites. The intraoral sites include edentulous ridges, tori, maxillary tuberosity or healing bony wound or extraction sites as well as mandibular ramus and chin area The extraoral sites most commonly used are iliac crest and ribs. ${ }^{17}$

Allogaft Graft: An allograft, formerly called as homograft, is a tissue graft between individuals of same species but of non-identical genetic composition. Allografts are grafts transferred between genetically dissimilar members of the same species. Allografts are probably incorporated into existing bone by a process similar to that of autogenous bone grafts, but proceed more slowly as a result of the absence of living cells. In animalstudies, allografts have been found to possess bonestimulating proteins and, consequently,osteoinductive properties. ${ }^{18}$ Use of any substitute graft material and the possibility of transfer of disease from donor to recipient as well as the presence and significance of immune responses to foreign antigens. Thus, bone banks accredited by responsible organizations exist for the purpose of supplying the surgeon with safe and effective bone tissue that is suitable for intended clinical application. The goals of bone banking are to preserve the physical integrity of the graft and the inductive protein, to reduce its immunogenicity, and to ensure sterility. ${ }^{19}$

Freeze-Dried Bone Allograft (FDBA): Freeze drying removes approximately $95 \%$ of the water from bone by a process of sublimation in a vacuum. Although freeze drying kills all cells, the morphology, solubility, and chemical integrity of the original specimen are maintained relatively intact. Freeze Dried Bone
Allograft (FDBA), but a number of synonyms exist (ground cortical, ground cancellous, freeze dried bone, etc.). Changes in particle size affects mainly handling characteristics, and many clinicians choose grafting materials on this basis. ${ }^{20}$

\section{Demineralized Freeze-Dried Bone Allograft} (DFDBA): Demineralized freeze-dried bone allograft (DFDBA) is nothing but freeze-dried bone allograft that is decalcified. The advantage is that, decalcification exposes the bone matrix proteins and hence induces osteogenesis. DFDBA is used because it contains bone morphogenetic protein (BMP), which induces new bone formation during the healing process.

Xenografts: Initially the term heterograft was used for these materials, but today they are termed as 'Xenografts' after the Greek word "xeno" which means foreign. Xenografts are surgical grafts transplanted between different species. Two sources of xenografts are commercially marketed as particulate bone replacement grafts in clinical practice bovine bone and natural coral. In order to render the animal bone non antigenic and safe for transplantation to human subjects, drastic chemical means were employed to remove the organic fraction of bone or markedly alter it. However this invariably produced an inert osseous product, which when placed in surgical defects has not been able to stimulate, even passively, new bone formation. ${ }^{21}$ Recently, new processing and purification method have been utilized which make it possible to remove all organic components from a bovine bone source and leave a non- organic bone matrix in an unchanged inorganic form.

Anorganic Bone: Anorganic bone is a bone from which organic material is extracted by means of ethylenediamine and sterilized by autoclaving. The use of this material was encouraged because of the protracted sequestration of the graft particles and slow resorption. ${ }^{22}$

Anorganic Bovine-Derived Bone Xenograft (BDX): Anorganic bovine bone is the hydroxylapatite "skeleton" that retains the macroporous and microporous structure of cortical and cancellous bone remaining after chemical or low-heat extraction of the organic component. For example- A unique regenerative product is PepGen $\mathrm{P}-15 \AA$, a calcined bovine bone $\left(1,100^{\circ} \mathrm{C}\right.$; hydroxyapatite) coated with a pentadecapeptide ( $\mathrm{P}$ 15 , a part of the sequence of collagen). In summary, bovine-derived hydroxypatite bone replacement grafts increase the available surface area that can act as an 
osteoconductive scaffold due to their porosity and have a mineral content comparable to that of human bone, allowing them to integrate with host bone. ${ }^{23}$

Anorganic Porcine-Derived Bone Xenograft: A natural replicate of autologous bone, OsteoBiol Gen-Osconserves the same intimate structures (matrix and porous form)and presents a high osteoconductive activity.

Alloplast: Alloplastic materials are synthetic, inorganic, biocompatible, and/or bioactive bone graft substitutes, which are claimed to promote bone healing through osteoconduction. The available alloplastic materials are Plaster of Paris, polymers, calcium carbonate, and ceramics. Ceramics can be classified into resorbable (e.g., tricalcium phosphate and resorbable HA) and nonresorbable (dense HA, porous HA, and bioglass).

Calcium Phosphate Ceramics: Larger number of ceramics are available, the calcium phosphate type has been of particular interest because of the close chemical and crystal resemblance of some of these materials to bone mineral. Commonly used calcium phosphate ceramics for periodontal regeneration are essentially of two types: The relatively nonresorbable $\mathrm{HA}(\mathrm{Ca} 10$ (PO4) $6(\mathrm{OH}) 2)$ or the resorbable tricalcium phosphates $(\mathrm{Ca} 3$ (PO4) 2).

Hydroxyapatite: The HA products used in periodontology are of two forms: A particulate nonresorbable ceramic form and a particulate resorbable nonceramic form. In controlled clinical studies, grafting of intrabony periodontal lesions with HA resulted in an attachment level gain of 1.1-3.3 mm which was greater as compared with nongrafted surgically debrided controls by Galgut et al. in 1992. ${ }^{24}$

Tricalcium Phosphate: Tricalcium phosphate has been shown to stimulate bone formation, and is comparable or in most cases superior in this regard to HA as described by Fetner et al. in $1994 .{ }^{25}$ It has been shown to stimulate bone formation to a greater extent than HA, but to a much lesser extent than bioglass as described by Wilson and Low in $1992 .{ }^{26}$ Cultured human fibroblasts have been demonstrated to attach readily to the surface of calcium phosphate ceramics. HA acts as an amphoteric ion exchanger. Selective accumulation of calcium and phosphate ion occurs as a consequence of the negative charges on the HA surface. This leads to the formation of more apatite and stimulates the formation of new bone.

Plaster of Paris: Plaster of Paris is biocompatible and porous, thereby allowing fluid exchange, which prevents flap necrosis. Plaster of Paris resorbs completely in 1 or 2 weeks. Its usefulness in human cases has not been proven.

Hard Tissue Replacement Polymer: HTR polymer is a nonresorbable, microporous biocompatible composite of poly-methylmethacrylate and polyhydroxyethylmethacrylate, a resorbable polylactic acid polymer. The polymer does not produce an inflammatory or immune response in contact with bone or soft tissue as described by Yukna in $1990 .^{27}$

Bio-Active Glasses and Ceramics: Bioglasses are composed of $\mathrm{Si}-\mathrm{CaO}-\mathrm{Na} 2 \mathrm{O}-\mathrm{P} 2 \mathrm{O} 5$ and are resorbable or not resorbable depending on the relative proportion of these components. When bioglasses are exposed to tissue fluids, a double layer of silica gel and calcium phosphate is formed on their surface. They have been extensively used in conjunction with medical and dental implants because they develop a layer of hydroxycarbonate-apatite on their surface following exposure to body fluids. When used on the surface of metal implants, this layer incorporates collagen fibrils and in this way produces a mechanically strong bond between implant and the adjacent bone surface as described by Hench and West in $1996 .^{28}$

New Innovations Pepgen P-15: It is a bone grafting material used to fill periodontal osseous defects that is composed of anorganic bovine-derived HA bone matrix combined with a synthetic cell-binding peptide.

Growth factor-enhanced matrix 21S Growth factorenhanced matrix (GEM) $21 \mathrm{~S}$ is a synthetic grafting system for bone and periodontal regeneration composed of a purified recombinant growth factor and a synthetic calcium phosphate matrix.

\section{Conclusion}

The use of bone graft in the field of dentistry worldwide has shown great acceptance by the patient. Though the current technologies can be considered as mature, many developments and improvements are made to mimic the biological properties of a human closely. Different kind of materials with different property has been used from way back and till date to treat different type of defects. 
Ethical Clearance: Taken From Subhatri Dental College Meerut.

\section{Source of Funding: Self}

Conflict of Interest: Nil

\section{References}

1. Sukumar S, Drízhal I. Bone grafts in periodontal therapy. Acta Medica (Hradec Kralove) 2008;51:203-7

2. Liu X, Shi B, Zheng Q, Li C. Alveolar bone grafting and cleft lip and palate. Plast Reconstr Surg2017;140:359-360.

3. Hegedus $\mathrm{Z}$. The rebuilding of the alveolar process by bone transplantation. Dent Cosmos 1923; 65:736.

4. Nabers CL, O'leary TJ. Autogenous bone transplants in the treatment of osseous defects. J Periodontol 1965;36:5-14.

5. Schallhorn RG, Hiatt WH, Boyce W. Iliac transplants in periodontal therapy. J Periodontol 1970;41:566-80

6. Dubok, V.A. Bioceramics-yesterday, today, tomorrow. Powder Metall Met Ceram. 2000; 39: 381-394.

7. Yukna RA. HTR polymer grafts in human periodontal osseous defects. I 6-month clinical results. J Periodontol 1990;61:633-42.

8. Richardson CR, Mellonig JT, Brunsvold MA, McDonnell HT, Cochran DL. Clinical evaluation of Bio-Oss: A bovine-derived xenograft for the treatment of periodontal osseous defects in humans. J Clin Periodontol 1999;26:421-8

9. Needleman IG, Worthington HV, Tucker RJ. Guided tissue regeneration for periodontal infrabony defects. Cochrane Database Syst Rev 2006;8:724-766

10. Kumar J, Jain V, Kishore S, Pal S. Journey of bone graft materials in periodontal therapy. J Dent and Allied Sci2016;3:30-34.

11. Mellonig JT, Bowers GM. Regenerating bone in clinical periodontics. J Am Dent Assoc 1990;121:497-502.

12. Gerald M, Bowers. Histologic Evaluation of New Attachment Apparatus Formation in Humans Part I. J Periodontol 1989;60:664-674.

13. Wang W, Yeung, K. Bone grafts and biomaterials
Medico-legal Update, January-March 2021, Vol. 21, No. 1977 substitutes for bone defect repair-Areview. Bio Mate 2017; 2: 224-247.

14. Stevenson S. Bone Grafting, Vet Clin North Am Small Anim Pract. 1999;29:1207-1219.

15. Stevenson S, Emery S, Goldberg V. Factors Affecting Bone Graft Incorporation. Clini Ortho 1996;324:66-74.

16. Auer J. Principles in Fracture Treatment, in Auer J (ed): Equine Surgery. Philadelphia W.B. Saunders 1992:812-832.

17. Froum SJ, Thaler R, Scopp IW, Stahl SS. Osseous autografts. Clinical responses to bone blend or hip marrow grafts. JPeriodontol 1975;46:515521.

18. Hallman M, Thor A. Bone substitutes and growth factors as an alternative complement to autogenous bone for grafting in implant dentistry. JPeriodontol 20002008;47:172-192.

19. Grover V, Kapoor A, Malhotra R, Sachdeva S. Bone allografts-A review of safety and efficacy. Indian J Dent Res2011;22:496-550.

20. Carlson ER, Marx RE, Buck RE. The potential for HIV transmission through Allogenic bone: A review of risks and safety. Oral Surg Oral Med Oral Pathol Oral Radiol Endod 1995;80:17-23.

21. Emmings, $F \mathrm{r}$ e d G. Chemically modified osseous material for restoration of bone defects. J Periodontol 20001974;45:385.

22. Krejci, Farha CF. Osseous grafting in periodontal therapy. Part I osseous graft material. Comp Cont Edu Dent 1987;10:95-100.

23. Krejci, C, Bissada, N, Farah C, Greenwell H. Clinical Evaluation of Porous and Nonporous Hydroxyapatite in the Treatment of Human Periodontal Bony Defects. J Periodontol 1987;58: 521-528.

24. Galgut PN, Waite IM, Brookshaw JD, Kingston CP. A 4-year controlled clinical study into the use of a ceramic hydroxylapatite implant material for the treatment of periodontal bone defects. J Clin Periodontol 1992;19:570-7

25. Fetner E, Martigan MS, Low SB. Periodontal repair using PerioGlas in non-human primates: Clinical and histologic observations. Compend Contin Educ Dent 1994;12:932-9.

26. Wilson J, Low SB. Bioactive ceramics for periodontal treatment: Comparative studies in the Patus monkey. JAppl Biomater 1992;3:123-9. 
27. Yukna RA. HTR polymer grafts in human periodontal osseous defects. I 6-month clinical results. J Periodontol 1990;61:633-42.
28. Hench LL, Splinter RJ, Allen WC, Greenlee TK $\mathrm{Jr}$. Bonding mechanism at the interface of ceramics prosthetic materials. J Biomed Mater Res Symp 1972;2:117-41. 